\title{
PRODUCCIÓN DE CONOCIMIENTO EN OCIO, RECREACIÓN Y TIEMPO LIBRE EN AMÉRICA LATINA
}

Recebido em: $27 / 11 / 2013$

Aceito em: 18/05/2014

\author{
José Fernando Tabares Fernández ${ }^{l}$ \\ Victor Alonso Molina Bedoya ${ }^{2}$ \\ Universidad de Antioquia \\ Medellín - Colombia
}

Julia Gerlero ${ }^{3}$

Pablo Bestrad ${ }^{4}$

Universidad Nacional del Comahue Neuquén - Neuquén Province - Argentina

Ari Lazzarotti Filho ${ }^{5}$

Universidade Federal de Goiás (UFG)

Goiânia - GO - Brasil

Cleber Dias ${ }^{6}$

Universidade Federal de Minas Gerais (UFMG)

Belo Horizonte $-\mathrm{MG}-$ Brasil

Elena Paz Morales $^{7}$

Universidad YMCA

Ciudad de México - Distrito Federal - México

Loreley Conde ${ }^{8}$

Eduardo Martínez ${ }^{9}$

Instituto Superior de Educación Física (UdelaR)

Uruguay

Eloy Altuve $e^{10}$

\footnotetext{
${ }^{1}$ Doctor en ocio y Potencial Humano de la Universidad de Deusto Bilbao-España.

${ }^{2}$ Doctor en Currículo, transversalidad y desarrollo sostenible Universidad de Valladolid.

${ }^{3}$ Magister Teorías y Políticas de la Recreación por la Universidad Nacional del Comahue y en Dirección de Proyectos de Ocio por la Universidad de Deusto -Bilbao-, España.

${ }^{4}$ Licenciado en Letras de la Universidad Nacional de Córdoba y Posgrado en Gestión Cultural por la Facultad de Economía de la Universidad Nacional de Córdoba.

${ }^{5}$ Doctor en Educación Física por la Universidad Federal de Santa Catarina.

${ }^{6}$ Doctor en Educación Física por la Universidad Estadual de Campinas.

${ }^{7}$ Maestría en recreación y administración del tiempo libre Profesora Escuela Politécnica del Ejército en Quito, Ecuador, Titular en la Maestría en Recreación y en la licenciatura en Administración del Tiempo Libre de la Universidad YMCA.

${ }^{8}$ Profesora de Educación Física.

${ }^{9}$ Profesor de Educación Física.
} 
RESUMEN: El presente artículo, primer resultado parcial de la investigación sobre ocio, recreación y tiempo libre en América Latina desarrollado por un equipo internacional, centra su atención en los diferentes asuntos que constituyen las condiciones de posibilidad generales para la producción de conocimiento en cada uno de los países participantes, es decir, Argentina, Uruguay, Brasil, Ecuador, Venezuela, Colombia Panamá y México. Asuntos como la formación de posgrado, los grupos de investigación, las publicaciones, los eventos, la colaboración entre pares tanto en el nivel nacional como regional, entre otros, son abordados para establecer tales condiciones generales en los diferentes países. Se hace evidente la diversidad de cada uno de los países en relación con el desarrollo de este campo de investigación.

PALAVRAS CHAVE: Epistemologia. Atividades de Lazer. Recreación. America Latina.

\section{MAKING KNOWLEDGE IN LEISURE, RECREATION AND FREE TIME IN LATIN AMERICA}

ABSTRACT: This article, first partial resulted from the research on recent scholar productions about leisure, recreation and free time in Latin America, doing by an international team, draws upon different issues related with social conditions of making knowledge in Argentina, Uruguay, Brazil, Venezuela, Colombia, Panamá and Mexico. Issues as post-graduation, research's groups, journals, congress and opportunities for scholar collaboration was approached for establishing the social conditions to making knowledge on recreation, leisure and free time in these countries. There is considerable diversity in the development of leisure studies among these countries.

KEYWORDS: Knowledge. Leisure Activities. Recreation. Latin America.

\section{Introducción}

La producción de conocimiento en el campo del ocio, la recreación y el tiempo

libre en América Latina, no ha sido tradicionalmente un tema de preocupación y

\footnotetext{
${ }^{10}$ Sociólogo y Licenciado en Educación, en Ciencias Sociales.

${ }^{11}$ Doctor en Educación por la Universidad Pedagógica Experimental Libertador de la República

Bolivariana de Venezuela.
} 
José Fernando T. Fernández, Produccion de Conocimiento en Ocio, Recreacion y Tiempo Libre... Victor Alonso M. Bedoya, Julia Gerlero,

Pablo Bestrad, Ari Lazzarotti Filho, Cleber Dias,

Elena Paz Morales, Loreley Conde, Eduardo Martínez,

Eloy Altuve e Alixon Reyes

discusión central entre los académicos e investigadores de la región. Entre algunas de las razones se pueden señalar la dependencia del campo a influencias extranjeras, la falta de una adecuada comunicación que impide conocer los desarrollos logrados en los diferentes países, la poca cultura de socialización de lo que se hace y se investiga más allá de las fronteras nacionales y el aún bajo nivel de producción de nuevo conocimiento en el campo de indagación, influenciado entre otras circunstancias, por una perspectiva instrumental, manifiesta en una sobrevaloración de las prácticas en detrimento de asuntos como la investigación y la generación de conocimiento propio.

Sin embargo, en la actualidad el asunto empieza a ubicarse como preocupación entre algunos investigadores y grupos de investigación. Las nuevas tendencias de la gestión del conocimiento, las exigencias a las que deben responder los investigadores, las universidades, las corporaciones e institutos de investigación hoy, además de la política de internacionalización, ponen la producción de conocimiento como relevante en la nueva estructura organizativa del trabajo y del consumo en la sociedad de la globalización o de la revolución capitalista, en cada uno de los países. Los diferentes niveles de desarrollo de cada uno de los países y la ausencia de una comunicación fluida entre estudiosos impiden conocer a fondo la situación actual del campo en la región latinoamericana. Empero, procesos conjuntos han sido adelantados por algunos grupos de investigadores de la región (cf. GOMES, et. al., 2009).

En este contexto, la pregunta por las condiciones de posibilidad para la generación de conocimiento, no es de fácil respuesta. Este artículo, en el marco de la investigación sobre la producción de conocimiento en ocio, recreación y tiempo libre en América Latina, que analiza la producción académica sobre estos asuntos entre 2000 y 2010, con la participación de investigadores e investigadoras de Argentina, Uruguay, 
estados de desarrollo de la investigación se han hecho visibles. Su identificación y tratamiento constituye un primer ejercicio que aportará información relevante para la potenciación del campo. Asuntos como las realidades particulares de cada país, el apoyo y valoración de la producción de conocimiento como política estatal, el desarrollo de la producción científica específica a partir de corpus que permitan establecer asuntos sobre intereses y tendencias; la existencia o no de procesos de formación de maestría y doctorado que actúen como condición de posibilidad para la formación de investigadores, los grupos de investigación existentes y ranqueados, sus intereses, temáticas y número de investigadores; los eventos académicos como escenarios de difusión y debate, y la existencia de revistas especializadas, podrían permitir un acercamiento a la situación actual de la temática en la región latinoamericana.

Para este momento de la investigación, tres categorías han sido fundamentales. En primer lugar, epistemología, que a la manera de Jaime Breilh (2002) se abordó como el conjunto de condiciones de posibilidad de lo que se puede decir, pensar y conocer en un momento histórico específico. En segundo lugar se encuentra la producción de conocimiento, que desde una perspectiva crítica, entiende que el análisis de la investigación es un acto de desarrollo cognitivo y al mismo tiempo un desafío ético y político. Por último se tiene la geopolítica conocimiento, como el reconocimiento del carácter hegemónico de la reproducción, difusión y uso del conocimiento. Para Walsh (2004):

\footnotetext{
La mayor consecuencia de la geopolítica del conocimiento es poder comprender que el conocimiento funciona como la economía: está organizado mediante centros de poder y regiones subordinadas- los centros del capital económico también son los centros del capital intelectual (p. 3).
} 
Categoría fundamental para el análisis de la producción de conocimiento en el campo en tanto permite comprender que el conocimiento no es abstracto, des-localizado ni des-incorporado. Por el contrario, tiene color, lugar, género y valor. Es decir, en la sociedad hegemónica, las formas de fabricación de conocimiento reproducen los patrones de la colonialidad del poder y derivan en la colonialidad epistémica; por la cual se instala tanto en el imaginario como en la práctica cotidiana, la idea de un conocimiento superior y legítimo, que procede de unos determinados lugares y de unas determinadas lenguas.

En esta dirección, preguntas como las siguientes orientan la indagación: ¿Qué pasa en nuestros países con la generación de conocimiento en el campo de los estudios del ocio? ¿Qué hace posible la condición de lugares productores de conocimiento y lugares receptores del mismo? ¿Qué se ha escrito sobre el tema del ocio? ¿Alrededor de qué objetos se produce conocimiento? ¿Qué problemas abordan los académicos en sus ejercicios de investigación? ¿Qué tipo de investigación se hace? ¿Qué discursos circulan y qué prácticas se promueven?

Interrogantes que han permitido concertar esta investigación. Así, el propósito de este primer documento de construcción colectiva, es presentar la situación actual de las condiciones de posibilidad del campo en cada uno de los países que participan de la investigación.

\section{Argentina}

La primera década del siglo XXI ha sido escenario de un incremento exponencial de las investigaciones sobre el tema en la Argentina, en relación con la década anterior. 
En referencia a los procesos de formación, existen tres carreras universitarias y terciarias en el campo de la recreación ${ }^{12}$; dos en el tema Tiempo Libre ${ }^{13}, 55$ en la Educación física y deporte y 171 en turismo, hotelería, administración de empresas turísticas, gestión del patrimonio y turismo sostenible. También existe un Instituto privado de 36 años ininterrumpidos, cuyo objeto de estudio es el Juego y la creatividad, además de varias instituciones municipales.

Las fuentes analizadas más concurridas son, en orden decreciente: tesis de posgrado, publicaciones de libros, artículos en revistas y, en último lugar, publicaciones en actas de congresos. Las tesis constituyen el 55\% del corpus analizado hasta el momento, los libros un 22\%, 14\% los artículos de libros y un 3\% los artículos de revistas y también 3\% los artículos de actas de congresos.

La publicación especializada con más trayectoria es la revista "Recreación" de la ciudad de Córdoba. Caracteriza la producción del país la baja cantidad de publicaciones. Es más, un número alto de artículos de libros, de revistas y de actas de congresos de profesionales argentinos se realizan en medios del exterior.

En un primer análisis de las fuentes, preliminar y exploratorio, se pueden destacar algunas conclusiones parciales. Sobresale la baja producción sobre los temas, la dispersión de la información, así como la escasa circulación de la misma. En el mismo sentido, se encuentra, que ante un objeto de estudio cuyo recorte no está bien delimitado, los abordajes se hacen desde otros campos disciplinares. Valga de ejemplo

\footnotetext{
${ }^{12}$ Maestría en Teoría y Políticas de la Recreación (Universidad Nacional del Comahue). Especialización Superior en Recreación y Tiempo Libre UCASAL Salta. Técnico Nacional en Recreación CABA Instituto Superior de Ciencias de la Salud

13 Licenciatura en Tiempo Libre para el deporte y el turismo Universidad Nacional de Cuyo. Especialización superior en Recreación y tiempo libre UCASAL
} 
que los estudiosos más cercanos a la producción teórica provienen de campos disciplinares de la arquitectura y las ciencias de la educación.

En un mapa de país, los centros de estudios que abordan con cierta tradición y continuidad los temas, son la región del Río de La Plata cuyos epicentros son capital Federal y Mar del Plata. En el caso de esta última las investigaciones vinculan al ocio y la recreación con la planificación y las políticas públicas relacionadas con la lógica del turismo. Para Capital Federal, el Estudio Inés Moreno, de más de tres décadas de acción, trabaja el tema de la recreación en múltiples perspectivas tales como la creatividad, los usos empresariales y terapéuticos. Para la región patagónica los abordajes versan sobre la teoría y las políticas de la recreación.

Como conclusión provisoria señalar que en el campo de la producción sobre el tema no existe una lógica que delimite el objeto de estudio y la tradición se encuentra en una etapa inicial aunque con perspectivas de crecimiento. Este estado de fragmentación teórica ha permitido apenas generar controversias propias de los campos ya constituidos en el ámbito de la pedagogía, la sociología, el consumo, refiriendo el tema "recreación" de manera subsidiaria.

Un rasgo común del corpus analizado es que la mayoría de los escritos tratan la práctica y en ese ejercicio para la explicación los autores recurren a otras disciplinas tales como la sociología y la pedagogía. Un elemento reiterativo es la relación de la recreación con el ámbito del aprendizaje. En el mismo orden abundan los estudios que analizan casos específicos (la recreación en un centro turístico, en un ámbito escolar, en un espacio determinado) y el tema del "juego" aparece incorporado al ámbito institucional educativo. 
Los eventos concernientes al tema de esta investigación no cuentan con una sistematicidad ni tradición marcada. Han comenzado a producirse encuentros con cierta frecuencia y continuidad pero a partir del año 2010, precisamente fuera del ámbito de la unidad temporal de análisis de este trabajo. ${ }^{14}$ En estos encuentros prima la referencia experiencial siendo escasa la confrontación teórica.

\section{Brasil}

La producción científica brasilera ha recibido un gran estímulo en los últimos años. Entre 2001 y 2011, el país saltó de la $17^{\mathrm{a}}$ a la $13^{\mathrm{a}}$ posición en el ranking de los países que más publican artículos en el mundo. Considerando solo la base Scimago, fueron más de 49.664 artículos en 2011 (RIGHETTI, 2013). La mayor parte de esas investigaciones se realizan en universidades públicas. Los recursos que las financian, del mismo modo, vienen de organismos públicos. El sector de la investigación científica y tecnológica aparece como uno de los diez sectores que más han recibido incentivo del gobierno brasilero.

Como resultado de todo esto, también en los llamados "estudios de ocio", el país conoce una producción bastante voluminosa. Son literalmente innumerables tesis, libros y artículos producidos todos los años. Una primera característica de esa producción es su dispersión. En Brasil, trabajos sobre el ocio se encuentran en memorias de diferentes congresos, en libros de varias editoriales y también en revistas diversas, de las más distintas áreas.

\footnotetext{
${ }^{14}$ Nos referimos a: Cumbre de Recreación en conmemoración al Bicentenario del Natalicio de Domingo Faustino Sarmiento, "Abrir la puerta de los derechos para ir a jugar-se" (SanJuan- 29 y 21 de Agosto del 2011); Cumbre de Recreación, Juego y Arte- Córdoba (1 y 2 de Octubre del 2011); $1^{\circ}$ Congreso Latinoamericano de Recreación: Encuentro de Educación, Juego y Arte (Salsipuedes - Córdoba 8 y 9 de octubre 2012).
} 
José Fernando T. Fernández, $\quad$ Produccion de Conocimiento en Ocio, Recreacion y Tiempo Libre... Victor Alonso M. Bedoya, Julia Gerlero,

Pablo Bestrad, Ari Lazzarotti Filho, Cleber Dias,

Elena Paz Morales, Loreley Conde, Eduardo Martínez,

Eloy Altuve e Alixon Reyes

El directorio de grupos de investigación del Consejo Nacional de Desarrollo

Científico y Tecnológico de Brasil (CNPq) registraba, en febrero de 2013, 230 grupos relacionados con el ocio (disponible en http/dgp.cnpq.br/buscaoperacional). Es un número importante, aunque no signifique que existan, de hecho, ese total de grupos efectivamente dedicados al ocio. La herramienta de búsqueda considera las palabras utilizadas en la descripción del grupo, en sus líneas de investigación o en trabajos publicados. Así, el uso de la palabra clave ocio por un grupo de investigación en cualquiera de esos niveles denunciaría algún tipo de relación. Solo un análisis más detallado, sin embargo, podría determinar con mayor precisión la pertinencia o impertinencia de considerar cada uno de esos grupos en el ámbito de actuación de los estudios de ocio.

De todos modos, cada una de esas fuentes reúne trabajos de alguna forma relevantes sobre la temática. Así, una tentativa de analizar la producción sobre el ocio en Brasil puede llevar a resultados significativamente diferentes, de acuerdo con el conjunto de informaciones seleccionadas. En efecto, cualquier ensayo de realizar una revisión de la producción académica brasilera sobre el ocio tiende a ser parcial e incompleta.

Una búsqueda con la palabra-clave "ocio" en Scielo (Scientific Electronic Library Online), por ejemplo, considerando simultáneamente todos los índices (títulos, autor y resumen) registró, en mayo de 2013, un total de 459 apariciones en 129 revistas (disponible en http://www.scielo.org/php/index.php). De este total, 332 son del área de las ciencias de la salud (72\%) y apenas 97 del área de las ciencias humanas (21\%). El resto se distribuye en pequeñas proporciones entre las ciencias sociales aplicadas 
(5.4\%), ciencias biológicas $(3 \%)$, ciencias agrícolas $(1.3 \%)$, ingeniería $(0,87 \%)$ y lingüística, letras y artes.

Aunque teniendo en cuenta que búsquedas muy amplias, como esta, frecuentemente pueden identificar trabajos que, en la práctica, no tienen relaciones explícitas con el asunto, sería correcto inferir de ese cuadro que el ocio en Brasil ha sido estudiado prioritariamente en el ámbito de las ciencias de la salud (medicina, enfermería, y salud colectiva por ejemplo).

La respuesta a esta pregunta depende en gran medida de lo que se entiende exactamente por "estudios del ocio". Aunque cualquiera de los trabajos de alguna forma relacionados con el ocio sean potencialmente útiles para investigadores interesados en el asunto, definiciones excesivamente exhaustivas tal vez deberían ser evitadas. Para fines analíticos, los "estudios de ocio" pueden ser definidos como un campo multidisciplinario de las ciencias sociales dedicado específicamente a analizar los modos como diferentes sociedades o grupos sociales ocupan y utilizan, en el pasado y en el presente, sus "tiempos libres", esto es, la forma como descansan, festejan, juegan, danzan, se divierten, en fin. Se trata por tanto, de una especialidad temática.

En este sentido, sería recomendable que un análisis sobre los "estudios del ocio" considerase trabajos que se ocupan explícitamente de prácticas de divertimiento, formas de descanso, o problemas relacionados con el uso social, de manera más general. Trabajos publicados en las memorias de eventos como el Seminário O Lazer em Debate o el Encontro Nacional de Recreação e Lazer (Enarel) serían, entonces, fuentes privilegiadas para el caso brasilero.

El Enarel, en formato sutilmente diferente al actual, surgió en 1989, en Brasilia, en una iniciativa liderada por un grupo de profesores de la región. Se trata de 
José Fernando T. Fernández, Produccion de Conocimiento en Ocio, Recreacion y Tiempo Libre... Victor Alonso M. Bedoya, Julia Gerlero,

Pablo Bestrad, Ari Lazzarotti Filho, Cleber Dias,

Elena Paz Morales, Loreley Conde, Eduardo Martínez,

Eloy Altuve e Alixon Reyes

uno de los mayores y principales encuentros dedicados al ocio en Brasil, reuniendo

profesionales, gestores y académicos. Según datos revelados por Marcelino (2010)

“[...] el público medio de cada edición del Enarel es superior a 500, llegando a sobrepasar el número de 1000 participantes” (p. 13).

La iniciativa nace con el nombre de Encuentro de profesionales del Ocio. El objetivo inicial, como el nombre ya sugería, era el de intentar crear un espacio para el debate sobre los desafíos de la actuación profesional en ocio. Pero esta orientación fue transformada ya en el segundo encuentro, abandonándose el nombre de encuentro de profesionales, apuntando a la ampliación de las posibilidades de participación de otros grupos de interesados.

Una inclinación más académica siempre estuvo presente en las ambiciones de la iniciativa. Tanto que los tres primeros eventos fueron organizados por el Departamento de Educación Física de la Universidad de Brasilia. En 1995, según lo señalado por Marcellino (2010), aumentó el “apoyo sistemático de universidades, como soporte en las comisiones científicas” (p. 14). A partir de 1997, como una demostración más en este sentido, las memorias del evento pasarán a ser regularmente publicadas, expresando preocupaciones por sistematizar las informaciones producidas sobre el ocio en Brasil. Parte de esas publicaciones ya fueron analizadas por otros investigadores, que la retomarán a fin de realizar una revisión de la producción dedicada al ocio en Brasil. (cf. MARCELLINO, et al., 2009; MARCELLINO, 2010; STOPPA, et. al, 2010; TEREZANI, D. et. al., 2010).

A pesar de eso, como resultado de la especialización creciente de los investigadores dedicados al ocio, el ENAREL, que siempre alimentó la ambición de reunir teoría y práctica, parece haber eliminado la importancia de propiamente 

evaluación de Marcellino (2010):

\begin{abstract}
En los últimos años se ha registrado el debilitamiento de los encuentros entre gestores, animadores, profesores e investigadores, con la reducción de sus días y horarios y la coincidencia con otras actividades; disminución del espacio para presentación de temas libres y posters; disminución del espacio para conferencias y mesas redondas; y el aumento del espacio para talleres. Esos hechos, llevarán a los organizadores a la presentación al ENAREL del 2009, de un documento firmado por las principales organizaciones y grupos de investigación del área, mostrando preocupación con relación a la dirección del evento y sus objetivos, que estarían siendo descaracterizados (p. 19).
\end{abstract}

En líneas generales, se puede decir que será esta la situación de todos los otros eventos, con excepción, tal vez, del Seminário O Lazer em Debate, claramente comprometido con hacer progresar las fronteras de la producción de conocimiento sobre el ocio, gracias, sobre todo, a una coalición relativamente amplia entre profesores y estudiantes de cursos de posgrado. Aun así hay límites.

La actual orientación de las políticas científicas brasileras privilegia sobre manera la publicación de trabajos en revistas, ya no en memorias de eventos. De esa manera, reaccionando y tratando de ajustarse a tales exigencias, la mayoría de los investigadores han canalizado sus principales producciones hacia revistas científicas, que se tornarán vehículos capitales para la difusión del conocimiento científico. Lo mismo en las ciencias humanas, en general, que tradicionalmente se sirven de libros para presentar los resultados de investigación, señala ya cambios en ese sentido. Según cálculos de Abel Packer (2011), en 2009, 49\% de todas las citaciones generadas por trabajos del área de ciencias humanas en Scielo Brasil, ya se interesaba por las revistas.

En ese contexto, la revista Licere, editada desde 1998 por la Universidad Federal de Minas Gerais (UFMG) se convierte en una fuente privilegiada para visualizar la producción sobre ocio en Brasil: única revista brasilera dedicada específicamente a 
publicar trabajos sobre ocio. Desde su creación, 36 fascículos han sido publicados, totalizando más de 280 artículos. No es gratuito que esfuerzos recientes para revisar la producción sobre ocio en Brasil, se hayan ocupado de analizar la revista. (cf. ALVES, et al., 2012; STOPPA, et al., 2013).

\section{Colombia}

En 1998, se instituye un dispositivo de gestión para el sector: El Plan Nacional de Recreación y su componente de investigación el Programa Estratégico de Investigación en Recreación PENIR (COLDEPORTES, 2009)

Entre los problemas que se identifican en ese momento se pueden mencionar la ausencia de una cultura del conocimiento, falta de capacidad para gestionar recursos para la investigación, ausencia de profesionales e investigadores con entrenamiento específico en recreación, que impide la generación de procesos rigurosos y sostenibles, y la ausencia de grupos de investigación y de mecanismos de difusión de resultados de investigación entre pares nacionales e internacionales y la ausencia de agendas regionales a largo plazo. Sin embargo, se señala la existencia de algunas Universidades, investigadores y grupos, que adelantan procesos aislados de investigación.

En referencia a los Programas de Posgrado, uno de los problemas identificados en el campo, es la ausencia de programas del nivel de maestría y doctorado. Solo existen programas de especialización. De los seis programas que aparecen registrados ante el Ministerio de Educación Nacional, solo dos están activos en la actualidad (CUADRO 1). Ante esta ausencia, las personas del campo se ven obligadas a adelantar sus procesos de formación avanzada, en campos como la educación, la salud y el desarrollo humano. Situación que conduce a la dispersión de producción, que se hace 
José Fernando T. Fernández, Produccion de Conocimiento en Ocio, Recreacion y Tiempo Libre...

Victor Alonso M. Bedoya, Julia Gerlero,

Pablo Bestrad, Ari Lazzarotti Filho, Cleber Dias,

Elena Paz Morales, Loreley Conde, Eduardo Martínez,

Eloy Altuve e Alixon Reyes

evidente en la configuración de corpus requeridos para ejercicios como el que

actualmente se adelanta.

Cuadro 1 - Programas de Posgrado en Ocio, recreación, Lúdica en Colombia (SNIES, 2013)

\begin{tabular}{|c|c|c|c|c|c|c|c|c|}
\hline $\mathbf{N}^{0}$ & Institución & Nombre posgrado & $\begin{array}{c}\text { Área de } \\
\text { Conocimiento }\end{array}$ & Nivel formación & Metodología & Duración & Título & $\begin{array}{r}\text { Situación } \\
\text { actual }\end{array}$ \\
\hline 1 & $\begin{array}{c}\text { Fundación } \\
\text { Universitaria } \\
\text { Juan de } \\
\text { Castellanos }\end{array}$ & $\begin{array}{l}\text { Especialización en } \\
\text { Lúdica Educativa }\end{array}$ & $\begin{array}{c}\text { Ciencias de la } \\
\text { Educación }\end{array}$ & Especialización & $\begin{array}{c}\text { Distancia } \\
\text { (Tradicional) }\end{array}$ & 2 Semestres & $\begin{array}{l}\text { Especialista en } \\
\text { Lúdica educativa }\end{array}$ & Activa \\
\hline 2 & $\begin{array}{l}\text { Universidad } \\
\text { de Pamplona }\end{array}$ & $\begin{array}{c}\text { Especialización en } \\
\text { Educación para la } \\
\text { Recreación } \\
\text { Comunitaria }\end{array}$ & $\begin{array}{c}\text { Ciencias de la } \\
\text { Educación }\end{array}$ & Especialización & Presencial & 2 Semestres & $\begin{array}{c}\text { Especialista en } \\
\text { Educaciónparala } \\
\text { recreación } \\
\text { Comunitaria }\end{array}$ & Inactiva \\
\hline 3 & $\begin{array}{c}\text { Escuela } \\
\text { Nacional del } \\
\text { Deporte }\end{array}$ & $\begin{array}{c}\text { Especialización en } \\
\text { Dirección y } \\
\text { Gestión de la } \\
\text { Recreación }\end{array}$ & $\begin{array}{l}\text { Economía, } \\
\text { administración, } \\
\text { contadurí, etc. }\end{array}$ & Especialización & Presencial & 2Semestres & $\begin{array}{l}\text { Especialista en } \\
\text { Dirección y } \\
\text { Gestión de la } \\
\text { Recreación }\end{array}$ & Inactiva \\
\hline 4 & $\begin{array}{c}\text { Fundación } \\
\text { Universitaria } \\
\text { Los } \\
\text { Libertadores }\end{array}$ & $\begin{array}{l}\text { Especialización en } \\
\text { Lúdica y } \\
\text { Recreación para el } \\
\text { Desarrollo Cultural } \\
\text { y Social }\end{array}$ & $\begin{array}{c}\text { Ciencias Sociales } \\
\text { y Humanas }\end{array}$ & Especialización & Presencial & 4 Trimestres & $\begin{array}{l}\text { Especialista en } \\
\text { Lúdica y } \\
\text { Recreación para el } \\
\text { Desarrollo } \\
\text { Cultural y Social }\end{array}$ & Inactiva \\
\hline 5 & $\begin{array}{c}\text { Fundación } \\
\text { Universitaria } \\
\text { Los } \\
\text { Libertadores }\end{array}$ & $\begin{array}{l}\text { Especialización en } \\
\text { Pedagogía de la } \\
\text { Recreación } \\
\text { musical para el } \\
\text { desarrollo Social y } \\
\text { Cultural }\end{array}$ & $\begin{array}{c}\text { Ciencias de la } \\
\text { Educación }\end{array}$ & Especialización & $\begin{array}{c}\text { Distancia } \\
\text { (Tradicional) }\end{array}$ & 2 semestres & $\begin{array}{c}\text { Especialista en } \\
\text { Pedagogía de la } \\
\text { Recreación } \\
\text { musical para el } \\
\text { desarrollo Social y } \\
\text { Cultural }\end{array}$ & Activa \\
\hline
\end{tabular}

Fuente: Construcción de los investigadores.

En referencia a los Grupos de investigación, en la plataforma de Colciencias, entidad responsable de la política de ciencia, tecnología e innovación en el país, aparecen 13 grupos registrados. Un grupo tiene como descriptor la palabra ocio, tres grupos incluyen el descriptor recreación, asociado a otros como actividad física, deportes, cultura física, educación física, muy propio de los programas que forman 
Victor Alonso M. Bedoya, Julia Gerlero,

Pablo Bestrad, Ari Lazzarotti Filho, Cleber Dias,

Elena Paz Morales, Loreley Conde, Eduardo Martínez,

Eloy Altuve e Alixon Reyes

profesionales en el país, y donde la recreación se comprende como un medio de la

educación física. Ocho de los grupos, se inscriben en el turismo, en ningún caso relacionado con el ocio, la recreación o el tiempo libre. Un grupo, aparece con el descriptor Animación Cultural. En general, se refleja una muy baja presencia de grupos de investigación inscritos en el campo de interés.

\section{Cuadro 2 Grupos de investigación registrados en Colciencias}

\begin{tabular}{|c|c|c|c|c|c|c|c|}
\hline \multirow[b]{2}{*}{ Descriptor } & \multirow[b]{2}{*}{ Nombre del Grupo } & \multirow[b]{2}{*}{ Total } & \multicolumn{5}{|c|}{$\begin{array}{c}\text { Áreas de los grupos con interés en los temas do ocio, recreación, } \\
\text { tiempo libre y lazer }\end{array}$} \\
\hline & & & Educación & $\begin{array}{l}\text { Ciencias } \\
\text { Humanas }\end{array}$ & $\begin{array}{l}\text { Ciencias } \\
\text { sociales } \\
\text { aplicadas }\end{array}$ & $\begin{array}{l}\text { Ciencias de } \\
\text { la salud }\end{array}$ & $\begin{array}{l}\text { Educación } \\
\text { Física }\end{array}$ \\
\hline Ocio & $\begin{array}{l}\text { Ocio, expresiones } \\
\text { motrices y sociedad }\end{array}$ & 1 & $\mathrm{X}$ & $\mathrm{X}$ & & & \\
\hline \multirow{3}{*}{ Recreación } & $\begin{array}{l}\text { Actividad física, } \\
\text { recreación y deporte }\end{array}$ & \multirow{3}{*}{3} & & & & $\mathrm{X}$ & $\mathrm{X}$ \\
\hline & $\begin{array}{l}\text { Ciencias sociales, } \\
\text { cultura física, deporte y } \\
\text { recreación. }\end{array}$ & & & & & $\mathrm{X}$ & $\mathrm{X}$ \\
\hline & $\begin{array}{l}\text { Epistemología e Historia } \\
\text { de la Educación Física, } \\
\text { la Recreación y el } \\
\text { Deporte -HIERDE- }\end{array}$ & & & & & $\mathrm{X}$ & $\mathrm{X}$ \\
\hline \multirow{8}{*}{ Turismo } & Turismo & \multirow{8}{*}{8} & & & $\mathrm{X}$ & & \\
\hline & Turismo y sociedad & & & & $\mathrm{X}$ & & \\
\hline & $\begin{array}{l}\text { Turismo, medio } \\
\text { ambiente y desarrollo }\end{array}$ & & & & $\mathrm{X}$ & & \\
\hline & $\begin{array}{l}\text { INCATUR Grupo de } \\
\text { Investigación en } \\
\text { Ciencias } \\
\text { Administrativas y } \\
\text { Turismo }\end{array}$ & & & & $\mathrm{X}$ & & \\
\hline & $\begin{array}{l}\text { Grupo de Investigación } \\
\text { Empresarial y Turístico }\end{array}$ & & & & $\mathrm{X}$ & & \\
\hline & $\begin{array}{l}\text { Desarrollo Turístico y } \\
\text { Regional }\end{array}$ & & & & $\mathrm{X}$ & & \\
\hline & $\begin{array}{l}\text { Grupo de Investigación } \\
\text { Impacto del Turismo } \\
\text { sexual GIFFI }\end{array}$ & & & & $\mathrm{X}$ & & \\
\hline & TURISMO & & & & $\mathrm{X}$ & & \\
\hline $\begin{array}{l}\text { Animación } \\
\text { Cultural }\end{array}$ & $\begin{array}{l}\text { Grupo de Investigación } \\
\text { para la Animación } \\
\text { Cultural "MUISUATA" }\end{array}$ & 1 & & & $\mathrm{X}$ & & \\
\hline \multicolumn{2}{|c|}{ Total consolidado } & 13 & 1 & 1 & 9 & 3 & 3 \\
\hline
\end{tabular}

Fuente: Construcción de los investigadores. 
El evento más representativo del campo en Colombia es el Congreso Nacional de Recreación. Aunque se realizaba más o menos desde 1990, es a partir de la quinta versión, que Funlibre asume la responsabilidad. En el marco del primer Plan Nacional de Recreación, se inicia con Simposios Nacionales de Recreación de investigación y formación; y de Vivencias y Gestión. Este ejercicio no genera dinámicas sectoriales incluyentes, pero si la organización y sistematización de documentos de informes presentados a los eventos.

Sobre revistas especializadas están los intentos de Cuadernos de Ocio, que entre 1997 y 2000 logra editar cinco números. Luego Cuadernos de Ocio y Sociedad, logra publicar tres números, con artículos resultados de investigación y aportes de académicos de otros países de América Latina. Igual sucede con la Revista Latinoamericana de Recreación con dos números en el 2011 y el 2012, que cuenta con la participación de varios académicos de diferentes países de la Región.

\section{México}

Las instituciones académicas del México que se consideraron para esta investigación, históricamente se han identificado como programas cuyo sentido central es la formación de profesionales en recreación, tiempo libre y ocio; relacionado con las investigaciones efectuadas en México por profesionales del campo de la recreación, el tiempo libre y el ocio de los programas de licenciatura en administración del tiempo libre y la maestría en recreación de la Universidad YMCA en la CD de México; y del programa de maestría en administración del tiempo libre y recreación de la Universidad Regional Miguel Hidalgo sede de Tampico, Tamaulipas. Otras iniciativas emplazadas en diferentes puntos del país, ofrecen programas del ámbito de la cultura física con la 
Elena Paz Morales, Loreley Conde, Eduardo Martínez,

Eloy Altuve e Alixon Reyes

integración de materias en recreación, como una oferta académica a nivel de

especialización, con una fuerte implicación y relación con la educación física, el entrenamiento deportivo y el deporte.

Lo anterior relaciona a 90 escuelas, institutos y universidades públicas y privadas que forman al talento humano en el ámbito de la educación física, entrenamiento deportivo, administración deportiva, actividad física y deporte, ciencias de la actividad física y entrenamiento deportivo entre otros programas académicos, incluyendo, claro, a la Universidad YMCA y a la URMH, según datos referidos por la Comisión de Formación, Capacitación, Certificación e Investigación (CFCCeI) del Sistema Nacional del Deporte (SINADE).

La complejidad de la construcción del estado del arte en México a partir de los documentos de tesis y trabajos finales de estos programas donde la recreación, el tiempo libre y el ocio son el objeto de estudio, aunque no fuera el objeto razón del programa académico, representaría la necesidad de una cobertura nacional, que en términos objetivos hoy resulta compleja. Sin embargo, es importante hacer referencia al significativo aporte multidisciplinar e interdisciplinar que representaría la información y análisis resultante de la investigación.

En el año 2008, Agustín Zamora Lamadrid presentó su tesis titulada Análisis teórico epistemológico de las corrientes de recreación, ocio y tiempo libre utilizadas en la URMH, para obtener el grado de maestro en Recreación y administración del tiempo libre de esta Universidad. El objetivo de este trabajo fue clasificar los trabajos de grado de acuerdo con el paradigma sobre el desarrollo teórico del ocio desde una visión interdisciplinaria y multidisciplinaria, al paradigma de gestión, administración de la 
En el año 2009, Lupe Aguilar en colaboración con Agustín Zamora publicaron la investigación sobre el desarrollo de la formación y la investigación en la recreación y el tiempo libre en México, teniendo como referencia los programas ofertados por la Universidad YMCA, la URMH, así como el programa técnico en recreación que imparte la Universidad Nacional Autónoma de México (UNAM) a través del Colegio de Ciencia y Humanidades. Es oportuno referir que esta investigación efectúa un análisis de las tesis presentadas por los candidatos a la maestría de la URMH (AGUILAR, 2009).

Estas dos investigaciones referidas, no presentan análisis alguno de tesis de grado del programa de recreación de la Universidad YMCA, dado que hasta últimas fechas se tienen registradas tesis para optar por el grado de maestro en recreación, que serán integradas en este estado del arte.

Por último y no menos importante, se integrarán al análisis los documentos resultados de los eventos organizados por la Academia de Administración del Tiempo Libre de la Ilustre y Benemérita Sociedad Mexicana de Geografía y Estadística, quien desde el año 2000 ha tenido la tarea de impulsar eventos académicos en beneficio del área de índole nacional e internacional con la colaboración de un número de instituciones académicas y gubernamentales.

\section{Venezuela}

El estado del arte en el campo de estudio de la recreación, ocio y tiempo libre muestra una cosecha interesante en el contexto de las comunidades del saber ya 
José Fernando T. Fernández, Produccion de Conocimiento en Ocio, Recreacion y Tiempo Libre... Victor Alonso M. Bedoya, Julia Gerlero,

Pablo Bestrad, Ari Lazzarotti Filho, Cleber Dias,

Elena Paz Morales, Loreley Conde, Eduardo Martínez,

Eloy Altuve e Alixon Reyes

constituidas en la República Bolivariana de Venezuela. Cosecha variada desde el mundo

disciplinar, que azuzada ahora por la complejidad y la diversidad de las representaciones sociales, la manifestación, inclusión y desarrollo de algunas experiencias investigativas y metodologías diversas (en especial aquellas de carácter cualitativo con sus correlatos biográficos, sociocríticos, etnográficos, fenomenológicos, aquellos provenientes de la teoría fundamentada, etc.), la iniciativa de creación de múltiples programas avanzados de formación en recreación por parte de profesionales entregados y comprometidos con el campo de estudios de la recreación, el surgimiento y desarrollo de movimientos populares organizados; la creación, continuidad, seguimiento, fortalecimiento y desarrollo de una política pública en materia de recreación como el Plan Nacional de Recreación para el Vivir Bien; la intención de conexión entre la universidad y la manifiesta voluntad política del Estado para el desarrollo de la recreación como política pública y como fenómeno sociocultural, tiene la posibilidad de enriquecerse y ampliarse en todas sus dimensiones.

En el campo de la producción de conocimiento en recreación, tiempo libre y ocio, tenemos presente el esfuerzo de instituciones como la Universidad Pedagógica Experimental Libertador (UPEL), el Instituto Panamericano de Educación Física (IPEF) en la Universidad del Zulia, el Instituto Nacional de Capacitación y Recreación de los Trabajadores (INCRET), el Instituto Técnico de Recreación Educativa (ITRE) en convenio con la Universidad Latinoamericana y del Caribe (ULAC), que desarrollan programas de formación e investigación en este campo. Sus correlatos se manifiestan en unidades y líneas de investigación específicas para su desarrollo, como por ejemplo, los Estudios en Recreación (Núcleo de Investigación en Pedagogía del Movimiento "Prof. Darwin Reyes", NIPEM). El desarrollo de eventos académicos para la formación y 
José Fernando T. Fernández, Produccion de Conocimiento en Ocio, Recreacion y Tiempo Libre... Victor Alonso M. Bedoya, Julia Gerlero,

Pablo Bestrad, Ari Lazzarotti Filho, Cleber Dias,

Elena Paz Morales, Loreley Conde, Eduardo Martínez,

Eloy Altuve e Alixon Reyes

divulgación de la investigación en el campo de la recreación, el tiempo libre y el ocio, ha sido favorecido por el esfuerzo de universidades como la UPEL (Maracay, Maturín y Barquisimeto, reconociendo en esta última sede el polo de mayor desarrollo), el ITREULAC. No obstante, el desarrollo de tales actividades no ha conducido aún a la sistematización deseada. Incluso, la revista RecreArte (NIPEM), nace recientemente como iniciativa de un grupo de investigadores dando sus primeros pasos a nivel nacional.

$\mathrm{Si}$ bien es cierto que lo anteriormente mencionado ofrece perspectivas alentadoras, es preciso decir que, no obstante tales aseveraciones, se impone la necesidad de reconocer que, el umbral existente entre lo que se ha hecho y lo que se ha investigado en recreación, es muy grande. Se ha hecho mucho en comparación con lo que se ha investigado, y aunque a juicio de los expertos, ésta es una realidad que se replica en otros campos del saber, no es menos cierto que en el campo de la recreación la brecha parece ser aún más amplia. La investigación en el campo de estudios de la recreación ha sido una de las hijas bastardas en el complejo marco de los estudios en las ciencias sociales en Venezuela. De alguna manera, ha sido la recreación un campo menospreciado en tanto "no es científico": respuesta de coordinadores de programas y centros de investigación.

Mientras tanto, sabemos que aún en lo investigado en Venezuela predomina una tendencia positivista e instrumental que ha servido, entre otras cosas, para legitimar y fortalecer un discurso y unas prácticas que se precian de ser humanistas y de hallar correspondencia en la realidad y en las necesidades de las personas, pero que en el fondo y tras un análisis exhaustivo, se develan insuficientes para reivindicar la dignidad humana, el desarrollo personal, la libertad y la cultura, como elementos a los que tributa 
la perspectiva de una recreación más asociada al consumo, al hacer cosas, a la actividad, al divertimento sin más, al esparcimiento. Incluso, alcanza para convertir en rentable la propaganda que entroniza a la recreación como posibilidad empresarial, más que como una verdadera dimensión de desarrollo humano.

Hay algunos otros elementos que a nuestro juicio condicionan el desarrollo del campo de estudios en recreación, ocio y tiempo libre en Venezuela, induciéndolo a perspectivas deterministas e inmediatistas. Entre varios de estos aspectos, están:

- Intereses personales y colectivos de ciertos grupos de investigación (creación de comunidades científicas que intentan imponer una especie de rectoría en el campo de estudios y en las formas diversas de investigar).

Minusvaloración de la recreación, ocio y tiempo libre como campo de estudios en comparación con otros campos del saber y el hacer humano (Educación Física, Deporte, Ciencias Aplicadas al Deporte, entre otras).

Incomprensión de las autoridades universitarias con respecto a la necesidad e importancia de ampliar los horizontes investigativos en el campo de estudios de la recreación, ocio y tiempo libre. Es probable que esta situación sea negada por algunos de los señalados, no obstante, la práctica gerencial lo revela (salvo contadas excepciones que bien vale la pena destacar).

Asunción de unas ideas de recreación emparentadas con el activismo (teoría de la actividad, teoría ergódica), el recreacionismo, entre otras corrientes.

Asunción de cortes metodológicos positivistas que reducen y limitan la investigación a ejercicios legitimadores (Ej.: una gran cantidad de trabajos en maestrías y doctorados se resumen a la implementación de programas 

recreativos realizados en alguna comunidad en función de la realización previa de una encuesta de intereses en el campo de las prácticas recreativas). Eso, a juicio de la comunidad científica que ha sido controlada y regulada por una cofradía que intenta copar los escenarios, "pareciera" darles la razón a quienes esgrimen que en efecto sí se toma en cuenta la necesidad y los deseos o intereses de las personas.

Se realizan eventos tendentes a tratar el tema de la recreación, la animación recreativa, el juego y otros, no obstante, la sistematización de tales experiencias es poca. Además de ello, poco se socializa lo que se produce en tales actividades.

La industria editorial en las universidades en Venezuela se ha visto disminuida progresivamente por diversas razones, aunque hay por supuesto excepciones.

Hay posibilidades de publicación de trabajos a través de los artículos en revistas especializadas, sin embargo, es poco el interés de parte de los investigadores por publicar. Hay pocas publicaciones de este tipo, y aunque no todas, muchos de quienes publican lo hacen debido a la presión o el requisito del ascenso universitario o la titulación. Es decir, no hay un interés real en la investigación y divulgación. Para llegar a esta conclusión, hemos entrevistado a estudiantes y profesores de postgrado (especialización, maestría y doctorado), profesores universitarios, directores y editores de revistas, coordinadores de programas de formación avanzado, etc.

\section{Uruguay}


El campo de la recreación, el tiempo libre y el ocio, se ha construido históricamente sobre la base del modelo sajón, que se instala a partir de la llegada de la YMCA en 1909. Esta corriente, que se asocia con el enfoque de la recreación reconocido como el recreacionismo, merece la asignación de un rol activo, en cuanto a la constitución del estado moderno uruguayo, en tanto dispositivo privilegiado de intervención del tiempo libre de la población, aspecto que entendemos central para lo que sería, en términos de Barrán (1989), el pasaje de una sensibilidad bárbara a una sensibilidad civilizada, impulsada por las fracciones dominantes de la clase hegemónica.

Desde sus inicios, y hasta nuestros días, este campo ha estado íntimamente relacionado con la educación física y otras instituciones vinculadas a la construcción de discursos en relación con los usos del tiempo libre. ${ }^{15}$ A partir de la segunda mitad del siglo XX, la realidad particular de Uruguay ha sido marcada por los procesos políticos vividos en un periodo dictatorial de carácter cívico militar (1973-1984) y la apertura democrática en el año 1985. Esa situación define aspectos muy importantes para la recreación y el juego. Es así, que este tipo de propuestas, son tomadas como herramientas educativas de carácter alternativo, ocupando espacios sociales relevantes junto a la educación popular.

En la actualidad el campo de la recreación, tiempo libre y ocio, ha tenido un gran desarrollo a nivel del mercado, son incontables las propuestas en donde aparecen estas categorías como un objeto más de consumo, aunque también existe una gama de propuestas educativas que incluyen a la recreación y otras prácticas culturales como

\footnotetext{
${ }_{15}$ Pudiendo identificar instituciones como la Comisión Nacional de Educación Física, los gobiernos Municipales, el Ministerio de Turismo y Deporte, la YMCA, las ONGs, Instituciones de educación formal e Instituciones privadas vinculadas a la educación física y el deporte.
} 
tomando a la recreación como estrategia para intervenir a nivel social.

En cuanto a la formación de los profesionales que actúan en el campo, son variadas e importantes las formaciones a nivel técnico, no siendo así en la formación superior específica. Algo que podemos formular a modo de hipótesis, es que existe un fuerte énfasis puesto en las actividades, donde la reflexión sobre la temática no presenta una producción sistemática.

En el país se pueden identificar tres instituciones vinculadas a la formación superior en recreación, en donde solo en una la formación es específica, representada por la Licenciatura en Recreación Educativa de la Universidad Católica del Uruguay; las otras dos refieren al campo de la Educación Física y están comprendidas por la Licenciatura en Educación Física de la Universidad de la República, y la Licenciatura en Educación Física, Recreación y Deportes del Instituto Universitario ACJ. ${ }^{16}$

El rastreo del desarrollo de la producción científica específica a partir del 2000 es identificado como un corpus con características más sistemático. A partir de la concreción del proyecto de investigación, el equipo responsable realizó reuniones periódicas con el objetivo de definir los posibles lugares donde recoger el corpus de documentos que presentan producción de conocimiento. Para ello, se propuso realizar un rastreo en bibliotecas de servicios universitarios, mediante el uso de la página BIUR (Bibliotecas de la Universidad de la República), a través de las palabras, ocio, recreación y tiempo libre. Así, se tuvo acceso a información primaria: título de los trabajos, autores, servicio de la U de la R y código de ubicación en la biblioteca. La

\footnotetext{
${ }_{16}$ A pesar de que los profesionales del campo provienen de variadas formaciones y algunas como las ciencias sociales y humanas son muy recurrentes en la formación de algunos actores claves del campo en Uruguay, entendemos que la presencia de cursos sobre recreación y tiempo libre, o la especificidad en cuanto a la temática de las carreras, hacen que sean en Uruguay las carreras más asociadas al campo.
} 
material identificado y comenzar con un primer análisis.

Asimismo, se concurrió a las ONG que trabajan en el temática, para indagar sobre la existencia de posibles publicaciones, donde se corrobora cierto grado de dispersión de los materiales que se han producido y de difícil acceso por no estar claramente identificados por quienes han participado en eventos o encuentros varios. Otra etapa consistió en realizar un rastreo primario en algún organismo público (Intendencia de Montevideo, Ministerio de Educación y Cultura) como forma de tener un primer paneo sobre los documentos publicados.

En forma paralela se dispuso realizar una indagatoria particularizada en la biblioteca del Instituto Superior de Educación Física, ya que por sus características en la formación del licenciado trabaja en la temática y en la producción de tesis finales de grado. Así también, se realizó un rastreo de materiales producidos por la tecnicatura en Tiempo Libre y Ocio dictada por la Universidad Católica, encontrando que los niveles de producción consisten en trabajos de carácter técnico y no académico. Por otra parte, se orientó la búsqueda en el centro de formación Asociación Cristiana de Jóvenes, institución encargada de la formación de licenciados en Educación Física a nivel privado, encontrándose una publicación en la "Revista universitaria de educación física y deportes" y el conocimiento de la producción de algunas tesis sobre la temática, como trabajo final de la carrera de grado.

Con respecto a la formación de posgrado, Uruguay no tiene procesos de formación de maestría y doctorado que actúen como condición de posibilidad para la formación de investigadores. 


\section{Consideraciones finales}

Un primer asunto que se hace evidente en el trabajo, es la diversidad de cada uno de los países, en relación con el desarrollo del campo. Contextos propios, que influyen en la configuración de condiciones de posibilidad diversas. Por ejemplo, Brasil aparece con un importante avance en las condiciones de posibilidad para la producción de conocimiento, en tanto que los demás países cuentan con muchas menos condiciones.

Países como Colombia y Uruguay no cuentan con propuestas de formación en los niveles de maestría y doctorado. La única revista seriada de la región dedicada al tema del ocio es Licere, de Brasil. Mientras que países como Colombia, con iniciativas intermitentes no cuenta con una propuesta regular, al igual que México y Uruguay. Venezuela cuenta que una revista de reciente creación y Argentina una revista cuyo objetico no es propiamente la difusión de nuevo conocimiento.

Lo corrido del siglo XXI, encuentra en los diferentes países condiciones propicias para el avance del campo, como se manifiesta explícitamente por países como Brasil, Argentina y Venezuela. El aumento de las publicaciones, los programas de posgraduación y los grupos de investigación, son evidencias del avance del campo en estos países.

Un asunto que se hace presente y que manifiestan los diferentes países latinoamericanos que hacen parte de esta investigación, es la dispersión de documentos e informes de trabajos hechos. Es decir no existe una sistematización sobre la producción en cada uno de los países. Igualmente sucede con la ausencia de un referente epistemológico que permita la delimitación del campo. Esto conduce a la existencia de múltiples acercamientos, condicionados por el campo de conocimiento 
José Fernando T. Fernández, Produccion de Conocimiento en Ocio, Recreacion y Tiempo Libre... Victor Alonso M. Bedoya, Julia Gerlero,

Pablo Bestrad, Ari Lazzarotti Filho, Cleber Dias,

Elena Paz Morales, Loreley Conde, Eduardo Martínez,

Eloy Altuve e Alixon Reyes

desde el que sea abordado (la salud, la pedagogía, educación, la sociología, la

psicología, la economía, el turismo, etc.). En líneas generales, esta situación, en estos aspectos particulares, no son diferentes de lo que pasa en el universo anglosajón (cf. ROWE, 2002).

En general, este trabajo permitió hacer evidente el estado actual de las condiciones de posibilidad para la producción de conocimiento en el campo en América Latina. Situación que debe ser potenciada a partir de los grupos de investigación, colectivos de académicos y universidades, buscando establecer agendas que posibiliten el desarrollo del campo en cada uno de los países, pero también con alianzas que permitan aprovechar las fortalezas de cada uno en beneficio de los demás para la consolidación de unas adecuadas condiciones de posibilidad para el desarrollo del campo en la Región.

\section{REFERÊNCIAS}

AGUILAR, Lupe. El desarollo de la formación y la investigación en la recreación y el tiempo libre en México. GOMES, C.; OSORIO, E.; PINTO, L.; ELIZALDE, R. (Org.) Lazer na América Latina/Tiempo libre, ocio y recreación en Latinoamérica. Belo Horizonte: Editora UFMG, 2009, p. 165-174.

ALVES, C., et. al. Lazer, políticas públicas não governamentais e estudos conceituais, na revista Licere. Revista da Educação Física, v. 23, p. 239-249, 2012. Disponible en: http://www.periodicos.uem.br/ojs/index.php/RevEducFis/article/view/14247. Visitado en: 2 ene. 2014.

BARÁN, J. Historia de la sensibilidad en el Uruguay. "La cultura 'bárbara': (18001860)". Montevideo: Ediciones de la Banda Oriental, 1989.

BREILH, J. Perspectivas políticas, sociales y éticas de la investigación en una era de barbarie. Revista da Escola de Enfermagem - USP, v. 36, n. 3, p. 210-221, 2002. 
José Fernando T. Fernández, $\quad$ Produccion de Conocimiento en Ocio, Recreacion y Tiempo Libre...

Victor Alonso M. Bedoya, Julia Gerlero,

Pablo Bestrad, Ari Lazzarotti Filho, Cleber Dias,

Elena Paz Morales, Loreley Conde, Eduardo Martínez,

Eloy Altuve e Alixon Reyes

COLDEPORTES COLOMBIA. Programa Estratégico Nacional de Investigación en

Recreación PENIR. Construcción colectiva de una agenda nacional de investigación, 2009. Disponible en: http://www.redcreacion.org/investigacion/penir.html. Visitado en: 10 dic. 2013.

GOMES, C. et al. (Org.) Lazer na América Latina/Tiempo libre, ocio y recreación en Latinoamérica. Belo Horizonte: Editora UFMG, 2009.

MARCELlinO, N. C. A. Maioridade do Enarel: análise do processo histórico de construção do Enarel. In: MARCELLINO, Nelson; ISAYAMA, Helder (Org.). Enarel: 21 anos de história. Brasília: Supernova Gráfica, 2010, p. 11-22.

. et al. Análise qualitativa dos trabalhos relacionados à temática "Lazer e políticas públicas", publicados nos Anais do ENAREL, de 1991 a 2008. Licere, Belo Horizonte, v. 12, n. 4, 2009. Disponible en: http://www.anima.eefd.ufrj.br/licere/sumario.html?ed=22. Visitado em: 15 ago. 2010.

PACKER, Abel. Os periódicos brasileiros e a comunicação da pesquisa nacional.

Revista USP, São Paulo, n. 89, p. 26-61, mar. / mai. 2011.

RIGHETTI, S. Produção científica do Brasil aumenta, mas qualidade cai. Folha de São Paulo, São Paulo, 22 de abril de 2013, n. 30.700, 2013, p. C8.

ROWE, David. Producing the Crisis: The State of Leisure Studies. Annals of Leisure Research, v. 5, issue 1, p. 1-13, 2002.

STOPPA, E. A. et al. A produção do conhecimento na área do lazer: uma análise sobre a revista Licere. Pensar a Prática, Goiânia, v. 16, n. 3, 2013. Disponible en: http://www.revistas.ufg.br/index.php/fef/article/view/17756. Visitado en: 2 ene. 2014.

et al. A produção do conhecimento na área do lazer: uma análise sobre as temáticas formação e atuação profissional nos Anais do ENAREL de 1997 a 2006. Licere, Belo Horizonte, UFMG, v. 13, n. 2, 2010. Disponible en: http://www.anima.eefd.ufrj.br/licere/pdf/licereV13N02_a3.pdf. Visitado en: 19 jun. 2013.

TEREZANI, D. et al. Lazer e meio ambiente: um estudo a partir dos anais do Encontro Nacional de Recreação e Lazer (ENAREL). Licere, Belo Horizonte, UFMG, v. 13, n. 1, 2010. Disponible en: http://www.anima.eefd.ufrj.br/licere/sumario.html?ed=23. Visitado en: 15 ago. 2010.

WALSH, C. Geopolíticas del conocimiento, interculturalidad y descolonializacion.

Boletín ICCI-ARY Rimay, año 6, n. 60, 1-5, mar. 2004.

ZAMORA, A. Análisis teórico epistemológico de las corrientes de recreación, ocio y tiempo libre utilizadas en la URMH. Tesis de Maestría Universidad Regional Miguel Hidalgo, México. 2008. (DSP). 
Elena Paz Morales, Loreley Conde, Eduardo Martínez,

Eloy Altuve e Alixon Reyes

\section{Endereço dos Autores:}

José Fernando Tabares Fernández

Calle 69 n. 43-49

Medellin - Colombia

Endereço Eletrônico: jfernando37@hotmail.com

Víctor Alonso Molina Bedoya

Carrera $57^{\mathrm{a}}$ n. 30B-04

Apartamento 201

La Florida - Bello

Antiowuis-Colombia

Endereço Eletrônico: vmolinacatios@yahoo.com

Julia Gerlero

Endereço Eletrônico: juliagerlero@hotmail.com

Pablo Bestrad

Endereço Eletrônico: pablobestard@gmail.com

Ari Lazzarotti Filho

Faculdade de Educação Física

Campus Samambaia

Caixa Postal 131

Goiânia - GO - 74001-970

Endereço Eletrônico: arilazzarotti@gmail.com

Cleber Dias

Av. Presidente Antônio Carlos 6627 - Pampulha

Belo Horizonte - MG - 31270-901

Endereço Eletrônico: cleberdiasufmg@gmail.com

Elena Paz Morales

Morena n. 506 Col de Valle

C.P. 03100

Delegación Benito Juárez

México Distrito Federal

Endereço Eletrônico: elenapazmorales@gmail.com

Loreley Conde

Endereço Eletrônico: loreley.conde@gmail.com

Eduardo Martínez

Endereço Eletrônico: eduardo.martinez293@gmail.com

Eloy Altuve

Edificio Quichua II, apartamento de la planta baja

Avenida 24 con calle 67 
José Fernando T. Fernández, $\quad$ Produccion de Conocimiento en Ocio, Recreacion y Tiempo Libre... Victor Alonso M. Bedoya, Julia Gerlero,

Pablo Bestrad, Ari Lazzarotti Filho, Cleber Dias,

Elena Paz Morales, Loreley Conde, Eduardo Martínez,

Eloy Altuve e Alixon Reyes

Parroquia Chiquiquira

Maracaibo - Estado Zulia - Venezuela

Endereço Eletrônico: eloyaltuve@hotmail.com

Alixon Reyes
La Puente, Carrera 13-A n. 21
Maturín, Estado Monagas
República Bolivariana de Venezuela
Endereço Eletrônico: alixdavid79@yahoo.com 